\title{
Using computer simulations and virtual reality to understand, design and optimize artificial water channels
}

\author{
X. Martinez ${ }^{12}$, A. Hardiagon' ${ }^{1,2}$, H. Santuz ${ }^{12}$, S. Murail ${ }^{3}$, M. Barboiu ${ }^{4}$, F. \\ Sterpone $^{1,2}$, M. Baaden ${ }^{1,2}$ \\ 1) CNRS, Université de Paris, UPR 9080, Laboratoire de Biochimie Théorique, 13 rue Pierre \\ et Marie Curie, F-75005, Paris, France \\ 2) Institut de Biologie Physico-Chimique-Fondation Edmond de Rotschild, PSL Research \\ University, Paris, France \\ 3) Université de Paris, CNRS, INSERM, Biologie Fonctionnelle et Adaptative UMR 8251, \\ Computational Modeling of Protein Ligand Interactions U1133, F-75205 Paris, France. \\ 4) Institut Europeen des Membranes, Adaptive Supramolecular Nanosystems Group, \\ Université de Montpellier, ENSCM, CNRS, Place Eugene Bataillon CC047, Montpellier, F- \\ 34095, France
}

In biology, metabolite transport across cell membranes occurs through natural channels and pores. Artificial ion-channel architectures represent potential mimics of natural ionic conduction. Many such systems were produced leading to a remarkable set of alternative artificial ion-channels. Far less advances were achieved in the area of synthetic biomimetic water channels, even though they could improve our understanding of the natural function of protein channels and may provide new strategies to generate highly selective, advanced water purification systems. Most realizations have used the selectivity components of natural protein channels embedded in artificial systems. Such biomolecules provide building blocks to constitute highly selective membrane-spanning water transport architectures. The simplification of such compounds, while preserving the high conduction activity of natural macromolecules, lead to fully synthetic artificial biomimetic channels. These simplified systems offer a particular chance to understand mechanistic and structural behaviors, providing rationales to engineer better artificial water-channels. Here we focus on computer simulations as a tool to complement experiment in understanding the properties of such systems with the aim to rationalize important concepts, design and optimize better compounds. Molecular dynamics simulations combined with advanced visual scrutiny thereof are central to such an approach. Novel technologies such as virtual reality headsets and stereoscopic large-scale display walls offer immersive collaborative insight into the complex mechanisms underlying artificial water channel function. 


\section{Introduction}

\section{Origins and brief overview}

Water is fundamental to life. It fulfills a variety of functions related to its complex dynamic properties at the supramolecular level [1]. Most physiological processes require selective traffic between a cell and its environment, where water is essential to enable molecular transport events [2]. Artificial ion-channels have been extensively studied with the expectation to mimic the ionic conduction along protein ion-channels through biological membranes [3-6]. Amazingly, until recently there has been almost no systematic progress in the area of water transport by Artificial Water Channels (AWCs) [7]. Before 2011, these artificial water pore systems, selectively transporting water against ions had not been described. We initiated this unexplored and highly innovative field with a series of studies described in [711]. Fig. 1 provides three examples of AWCs.

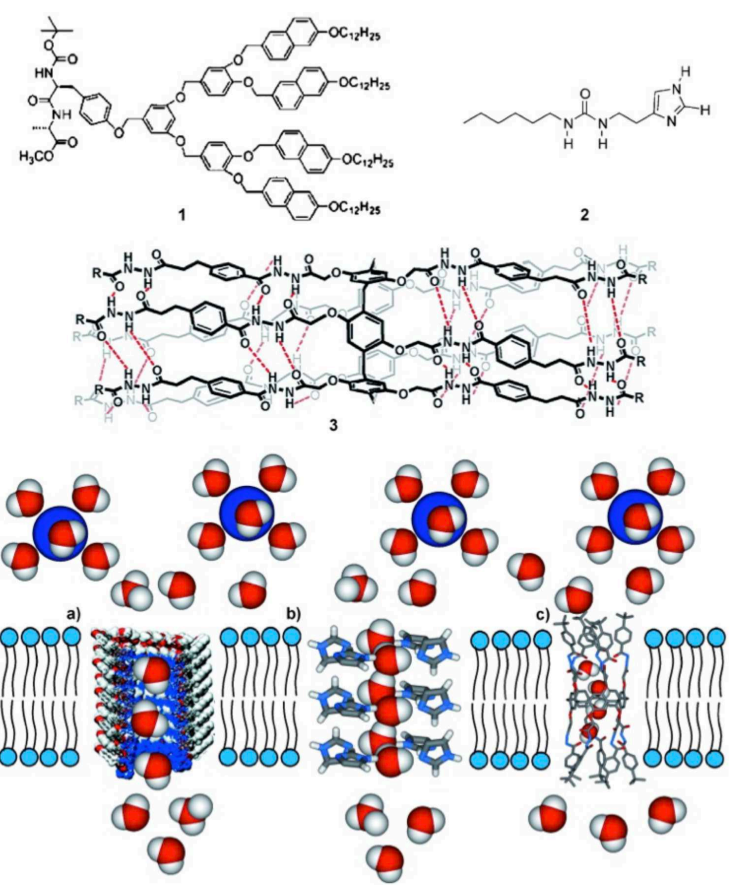

Fig. 1. Selection of artificial water channel systems: a) helical dendritic dipeptides, compound 1 ; $[12,13]$ b) oriented dipolar water wires within compound 2 chiral I-quartet assemblies [7-9] that selectively transport water and protons against ions; c) hydrazide-pilar[5]arene compound $\mathbf{3}$, [14-17] as single channels. Neutral and protonated water molecules are depicted as red and white CPK models. Blue spheres symbolize hydrated cations, unable to penetrate the bilayer membrane. Figure reproduced with permission from [8]. 
Since then, many other groups joined this adventure and many works have been published including a feature paper highlighting the developments of the field during the last 5 years [18]. Important studies of AWCs including molecular dynam ics (MD) simulations have been pioneered by several groups around the world, notably in the USA, China, Singapore, Hong-Kong, etc.

Molecular modeling approaches - in particular molecular dynamics in explicit solvent and membrane environments - constitute a pivotal tool to extend the methodology, providing insight at a molecular scale as described in [19-21]. Moving up in scales, the development of multi-scale approaches to describe hydrodynamics in molecular systems has the potential to provide new stimulus for water channel modeling $[22,23]$.

\section{Artificial water channels are conceptually important}

In contrast to isotropic spherical ions, dipolar water molecules shed light on multiple oriented interactions with biological environments. The overall orientation of assembled water dipoles is crucial for the regulation of the selective recognition, exchange and transport of charged biocomponents across biological barriers. For example, the electrochemical potential along the Aquaporin-Z Aqp-channel is governed by encapsulated single-file water wires of opposite dipolar orientation, selectively allowing rapid water diffusion, while protons and ions are excluded [24-28]. In comparison to classical polymeric systems, the water-permeability of AqpZ incorporated in polymeric vesicles is at least one order of magnitude larger [26]. One-dimensional water wires in particular have attracted broad interest with selective proton gating being the key function of the Influenza A M2 proton channel $[12,13]$. Entrapped water clusters within complex structures are a key element intervening in a variety of fundamental processes such as water structuration related to water-water or water-host-matrix interactions under confined conditions [47], selective transport of small metabolites [52], and in context of dynamic diffusion phenomena [14-17] of water molecules across the channels [17, 48]. So far, only a few artificial hydrophobic [29], hydrophilic [7-9] or amphiphilic pores $[30,31]$ have been designed to selectively and efficiently transport water through bilayer membranes. The molecular-scale hydrodynamics of water through the channel will depend on channel-water as well as water-water interactions, and on the in-pore water electrostatic dipolar profile within the channel. These molecular determinants can be controlled based on rational insight into AWC properties at the atomic scale. 


\section{Major insights are gained from molecular simulations}

In particular concerning molecular-scale processes, atomistic as well as simplified coarse-grained or meso-scale simulations have been pivotal to our understanding of key concepts of water transport in biological and synthetic systems. For instance, seminal work based on molecular dynamics simulations of Aquaporin channels has substantially contributed to understand molecular mechanisms responsible for their efficiency and selectivity [25, 28, 32]. General features of biological pores were studied by Beckstein and Sansom [33, 34], as well as others, in model systems of decorated simplified ion channel pores, providing rationales for designing improved channels. Another model system of great importance concerns the study of water transport in carbon nanotubes [35-37]. These few examples among many show that molecular-level insights are key to our understanding and can be achieved through molecular dynamics type simulations acting as a computational microscope. A few studies of artificial water channels including MD simulations have appeared recently and a Faraday Discussion documents the current status in the field [38-42]. The present discussion will firmly build on such simulation approaches to guide and optimize experimental design.

\section{Now and tomorrow in this newborn field}

Despite the tremendous imagination of synthetic chemists to produce sophisticated architectures confining water clusters, most strategies to transport water selectively have been inspired by the natural aquaporin scaffold as selectivity components, embedded in diverse arrays of bio-assisted artificial systems. The highly selective transport of water in natural systems is occurring along Aquaporin (AQP) channels known for their fast transport rates $\left(\sim 10^{*}-10^{\circ}\right.$ water molecules/s/channel), and the perfect rejection of ions and protons. Aquaporin water channels are composed of an hourglass structure with a narrowest constriction of $2.8 \AA$. Each water molecule in AQP water channels forms one hydrogen-bond with the inner wall of the protein and one with an adjacent water molecule. It is of particular interest that the dipolar alignment of water molecules imposed by the water-pore and water-water interactions can control the water $v s$. proton transport selectivity of AQPs. These discoveries have inspired the incorporation of AQPs into artificial membranes for desalination and water-purification applications. These results on the combination of natural proteins with artificial lipid matrices, have demonstrated that natural biomolecules can be used as building blocks for the construction of membranes for water transport. However, their large scale application is not yet possible, because of the high costs of AQP production, their low stability and practical synthetic constraints of membrane fabrication processes.

A possible way forward is to replace AQPs with synthetic channels, a major motivation for our work on AWCs. Recent advancements have made it possible to syn- 
thesize artificial water channels featuring efficient, yet selective transport of water based on size exclusion and molecular interactions, such as hydrogen-bonding. Given their completely artificial nature, these AWCs exhibit the following potential advantages:

- Synthetic AWCs that are easily tuneable and may be chemically optimized for desirable properties;

- AWCs can be prepared on a large scale at low cost;

- Engineering processing without bio-related steps becomes more simple and reproducible, and they can be easily immobilized in membrane-like supports in a scalable manner;

- AWCs should also be more robust and stable with a longer lifetime after incorporation into membranes for water separation and purification.

However, currently, the research on AWCs is focused mainly on the synthesis and characterization in terms of its water transport property and solute rejection. More focus should be turned on integrating the synthetic channels with a membrane for water separation and purification. Given the importance of developing a highly efficient membrane desalination technology, the potential of synthetic water channels and the knowledge gap for integration of synthetic water/ion channels into membranes, it is envisaged that development of synthetic channel-based biomimetic membranes with enhanced performance compared to current membranes is a challenging goal. It will require to provide answers to complicated biological scenarios related to water pores and to develop bio-inspired desalination processes that could use the non-existing all-made artificial synthetic materials that mimic the mechanisms of natural desalinators. Computational approaches can guide these endeavors.

\section{From Aquaporin to I-quartet channels}

\section{Learning from biological water-channels as sources of inspiration}

Potassium channel KcsA K+ [43], Aquaporin-AQP, [24-28], Influenza A M2M2IA, [12, 13] or Gramicidin-GA, [44] are well known, non-exclusive examples of proteins in which ions, water molecules and protons are envisioned to diffuse along water filled pores. It is worth to note that most protein channels share some recurrent structural aspects. For instance many are self-assembled from multiple subunits within the trans-membrane domains. These protein subunits are packed around a water filled pore, a crucial element in the functional conformation of these channels. The narrowest region of the pore usually determinesd the selectivity of these molecular channels, showing gating behaviors generated by the structural 
motion of the external subunits in response to voltage, ligand, $\mathrm{pH}$ or other external stimuli. The interactions between the constitutive components of the pore with water (most often a one-dimensional water wire) or with ionic species will impose a specific net dipolar moment controlling the translocation within the narrow region of the pore. Despite a wealth of experimental data on structural details of protein channels, major issues still need to be resolved and understood at the atomic level. For example, in Aquaporins, once passing the center of the pore occupied by a water molecule, the water molecules undergo a flip-like opposite orientation in the AQP channel. This inversion causes the inversion of the net dipolar moment and hinders proton transport. Therefore, the structure and alignment of the water wires within the synthetic pores may be important in the design of artificial pores creating (pumping) or collapsing (gating) ionic gradients throughout the membrane.

However there are many challenges to use the AQP scaffold as selective transporters related to their:

- Long term stability, which is unclear

- Unconventional processing requirements - use of detergent and specialized aqueous self-assembly

- Production - membrane proteins are challenging to mass produce

Other well-known natural ion-channels jointly transporting water and cations are of primordial inspiration for the design of the artificial channels. For example the dipolar structure of Gramicidin A-GA, helps to overcome the high energy barrier of water and ion translocations across the cell membrane, both being synergistically transported sharing one pathway [44]. Particularly interesting, the dipolar alignment of water molecules imposed by the pore structure can control the ionic conduction, including the translocation of protons via the inner-pore water wires. This oriented single-file water wire - as for the aquaporin pore - imposes a net dipole moment which is particularly influenced by the presence of the ions inside the channel. Depending on the position of the ion within the channel, two linear chains of water molecules of opposite orientation are determining the net dipole potential which is zero on average when the ion is in the middle of the channel. Similarly, the water molecules permeate through the KcsA K channel, together with the $\mathrm{K}+$ ions in a concerted way [43]. Interestingly, a $\mathrm{K}+$ depleted KcsA $\mathrm{K}$. channel is changing its initial conformation to allow water transport 20 times faster than one dimensional bulk diffusion of water [45]. On the same principle, the proton selectivity and low-pH gating properties are key functions of the M2 Influenza A - M2IA proton channel $[12,13]$. Although there is some variability to set off the mechanisms, many structural features are related to the (His" $)_{4}$ selectivity filter [46].

The encapsulation of water clusters in artificial constructs enables the exploration of the collective behaviors of water in conditions very close to biological systems where the confined molecules may be at the limit between solid and liquid states [14-17, 47-51]. One-dimensional water-wires in particular have generated much interest. Their structural features similar to water-clusters present inside the pro- 
tein channels were closely examined. Self-assembled tubular supramolecular architectures, mutually stabilized by strong non-covalent bonds and encapsulating inner water wires have provided excellent reasons to be considered as valuable models for biomimetic water/proton-channel systems [14-16, 49]. However, most of these structures are highly polar and it remains complicated to achieve their inclusion in hydrophobic bilayer membranes. All these non-exhaustive examples are relating specific features of the self-assembly behaviors of water clusters under confined conditions, toward systems of increasing functional complexity.

Understanding the molecular-scale dynamics of water clusters confined in such structurally simple artificial channels is of fundamental and crucial relevance for the next developments of functional AWCs. The mechanisms of water transport through aquapores are related to structural behaviors of water clusters entering the channels which lose or change part of the H-bonds connecting the water molecules, depending on the hydrophobic or hydrophilic nature of the channel [53]. Spontaneous and continuous diffusion of a one-dimensional ordered water chain in interaction or not with the surrounding aquapore is related to molecular interactions with the inner pore surface and diameter, dipolar water orientation.

With all these features in mind we can observe that natural water channels show a variety of sizes and different single-file water net dipole orientations that are directly connected to their specific function. They are selective toward other permeants (ions, protons, molecules) depending strongly on the channel structure and dimensionality, channel-water affinity, the average pore occupancy and the dynamics of the permeating water.

\section{Biomimetic artificial water channels}

Parallel to the investigations on natural water-channels the adventure of AWCs is just starting. Like in natural channels, the water transport will depend on key interactions, such as channel-water, water-water and electrostatics guided by the water in-pore electrostatic dipolar profile within the channel. Reproducing the complex interaction superstructures of proteins is an essential goal. A valid approach is to mimic key parts of active filters, as ingredients to provide chemical selectivity, in order to gain insight into the dynamics of orientating water dipoles. Particularly their role for ions/protons pumping along the channel has to be understood. Going from complex natural to simpler artificial water-channel constructs, we would have the chance to better comprehend mechanistic and structural behaviors of such interactions. Such knowledge may unlock the door to the novel interactive design of water-channels, paralleling that of biomolecular systems. Different synthetic building blocks have been reported until now to generate such systems: water was shown to transport through hydrophilic, hydrophobic or hybrid hydrophobic/hydrophilic channels as briefly summarized in Table 1 . 
Table 1. Compounds used as molecular building blocks to create artificial water channels [38]

\begin{tabular}{|c|c|c|}
\hline Compound & Nature of the channel & $\begin{array}{l}\text { Net Permeability/ Selectivity/ } \\
\text { Single channel permeability }\end{array}$ \\
\hline I-quartet Channels, $\mathbf{2}$ & \multirow{2}{*}{$\begin{array}{l}\text { Hydrophilic H-bonded imidaz- } \\
\text { ole channel }(2.6 \AA) \text { and hydro- } \\
\text { phobic peripheral alkyl chains }\end{array}$} & \multirow{2}{*}{$\begin{array}{l}1.0-4.0 \mu \mathrm{m} / \mathrm{s} \text { (shrinking mode) / } \\
\text { high selectivity for water reject } \\
\text { all ions except protons } / 1.5 \times 106 \\
\text { water molecules } / \mathrm{s} / \text { channel }\end{array}$} \\
\hline Alkylureidoimidazoles & & \\
\hline Aquaporins - AQPs & $\begin{array}{l}\text { Natural hourglass structure of } \\
\text { the pore, with a narrowest con- } \\
\text { striction of } 2.8 \AA\end{array}$ & $\begin{array}{l}\text { High water selectivity. Perfect } \\
\text { rejection of ions and protons / } \\
4 \times 109 \text { water mole- } \\
\text { cules/s/channel }\end{array}$ \\
\hline Aquafoldamers & $\begin{array}{l}\text { Hydrophilic helical channel } \\
(2.8 \AA) \text { via } \pi-\pi \text { stacking of aro- } \\
\text { matic units }\end{array}$ & $\begin{array}{l}\text { No permeability reported/ high } \\
\text { selectivity for water, reject all } \\
\text { ions except protons }\end{array}$ \\
\hline $\begin{array}{l}\text { Bola-amphiphile bis-triazole } \\
\text { (TCT), T-channels }\end{array}$ & $\begin{array}{l}\text { Self-assembled helical pores } \\
(\sim 2.5-4 \AA \AA) \text {; double helical chan- } \\
\text { nels double net-dipolar orienta- } \\
\text { tion }\end{array}$ & $\begin{array}{l}\text { No permeability reported for wa- } \\
\text { ter / enhanced conduction states } \\
\text { for alkali cations and for protons }\end{array}$ \\
\hline $\begin{array}{l}\text { Tubular pores formed via } \\
\text { self-assembly of arylene- } \\
\text { ethynylene macrocycle }\end{array}$ & $\begin{array}{l}\text { Hydropobic tubular channel } \\
(6.4 \AA) \text { via } \pi-\pi \text { stacking of ar- } \\
\text { omatic units and H-bonding of } \\
\text { peptide arms }\end{array}$ & $\begin{array}{l}51 \mu \mathrm{m} / \mathrm{s} / \text { no selectivity for water, } \\
\text { high conduction for } \mathrm{K} \cdot \text { and pro- } \\
\text { tons } / 4.9 \times 10^{\prime} \text { water mole- } \\
\text { cules/s/channel }\end{array}$ \\
\hline $\begin{array}{l}\text { Peptide appended Pil- } \\
\operatorname{lar}[5] \text { arenes, PAPs }\end{array}$ & $\begin{array}{l}\text { Hybrid hydrophobic/hydro- } \\
\text { philic cylindrical }(\sim 5 \AA ̊ ㇒) \text { uni- } \\
\text { molecular systems. }\end{array}$ & $\begin{array}{l}30 \mu \mathrm{m} / \mathrm{s} \text { (swelling mode, good } \\
\text { conduction for alkali cations } 3.5 \\
10 \text { (swelling mode) and } 3.7 \times 10 \\
\text { water } / \mathrm{s} / \text { channel (shrinking) }\end{array}$ \\
\hline $\begin{array}{l}\text { Hydrazide appended Pil- } \\
\text { lar[5]arenes, PAHs } 3\end{array}$ & $\begin{array}{l}\text { Hybrid hydrophobic/ hydro- } \\
\text { philic cylindrical ( } 6.5 \AA ̊ ̊) \\
\text { molecular systems }\end{array}$ & $\begin{array}{l}8.6 \times 10 \mu \mathrm{m} / \mathrm{s} / \text { no water selec- } \\
\text { tivity, good alkali cation conduc- } \\
\text { tion, but not for protons / } 40 \text { wa- } \\
\text { ter molecules/s/channel }\end{array}$ \\
\hline $\begin{array}{l}\text { Dendritic dipeptide, }(4-3,4- \\
\text { 3,5)-12G2-CH }{ }_{2}-B o c-L-T y r- \\
\text { L-Ala-OMe, } 1\end{array}$ & $\begin{array}{l}\text { Hydrophobic H-bonded central } \\
\text { pore }(14.5 \AA) \text {; stacked dendritic } \\
\text { periphery }\end{array}$ & $\begin{array}{l}\text { No permeability reported / water } \\
\text { against ions except protons }\end{array}$ \\
\hline Carbon Nanotubes- CTNs & $\begin{array}{l}\text { The estimated diameter of } \\
\text { CTNs }(12,12) \text { is } 16 \AA\end{array}$ & $\begin{array}{l}\text { No ion rejection: stable and high } \\
\text { permeability / } 9 \times 10 " \text { water mole- } \\
\text { cules/s/channel }\end{array}$ \\
\hline
\end{tabular}

Compounds are approximately ordered according to the channel diameter

The water diffusion and facilitated transport of protons excluding cationic and anionic transport through bilayer membranes incorporating all-artificial water channels have been reported for the first time by Percec et al. [29]. The dendritic dipeptides self-assemble via enhanced peripheral $\pi$-stacking to form stable cylindrical helical pores (14.5 $\AA$ in diameter), of reasonable thermal stability. Moreover they selectively transport water molecules against ions through selfassembled hydrophobic nanotubes stable in phospholipid membranes. The ionexclusion phenomena are based on hydrophobic effects which appear to be very important. These pores are envisioned to be the first artificial "primitive aqua- 
porins" which transport water but do not reject protons. Next, the significant contribution by $\mathrm{Hu}$ et al. [30, 31] shows that polydrazide-pillar[5] arenes tubular structures can be used as single-molecular water channels in bilayer membranes. The transport mechanism is strongly dependent on the length of the former components: the shortest pillar[5]arenes-tetraester, induce the formation of water wires within the stacked molecular cylinders and can successfully be used to translocate protons via bilayer membranes. The longer poly-hydrazide-pillar[5] arene compounds differently to shorter ones, present alternating hydrophobic/hydrophilic structural domains along their cylindrical structure which disrupt the formation of water wires within the inner channel core and thus block the proton flux along the discontinuous water phases. Differently to AQP, in which the control of the water/proton translocation is based on reverse-flip net-dipolar profiles within the channel [24], other channels may relate their selectivity to the structural disruption of water-wires.

\section{Imidazole I-quartet channels}

We reported [7-9] that imidazole I-quartet channels can be mutually stabilized by inner water-wires, reminiscent of the oriented water single-file columns observed for AQP and ion-charged GA natural pores. In addition, the I-quartet may be considered as the representative water-quartet superstructure, reminiscent of that of the most known Guanosine-G-quartet for cations (see Fig. 2 for a comparison). The I-quartet superstructures are stable in solid state and within bilayers leading to functional water-channels. These systems show total ion rejection and the ionexclusion phenomena are based on dimensional steric effects whereas hydrophobic and hydrodynamic effects appear to be less important.

As observed in aquaporin channels, the confined water wires form one hydrogen bond with the inner wall of the assembled I-quartet scaffold and another one with an adjacent water molecule. Furthermore, the water molecules embrace a unique dipolar orientation thereby preserving the overall electrochemical dipolar potential along the channel. These findings strongly support the idea that water molecules and protons can permeate across the lipid bilayer through pores formed by Iquartet aggregates. These I-quartet constructs provide good reasons to consider that their supramolecular chirality and water induced polarization (unique dipolar orientation) within the channels may be strongly associated [20]. Chiral surfaces are determinant to a more extended scale in asymmetric tissue morphogenesis.

Water is an electrically dipolar molecule and an exceptional (bio)lubricant; it can simply read the spatial information of the asymmetric chiral superstructures to generate asymmetric dipolar-wires of dynamic behaviors. Within this context, chiral synthetic pores of $2.8 \AA$ diameter (like the AQP natural ones!) orienting the dipolar water wires within the channels, give rise to novel strategies to mimic the protein-channel machinery. I-quartet channels offer an exciting opportunity to en- 
capsulate water-clusters in confined chiral space and offer a chance to explore water behaviors very close to pore-confined biological water, exhibiting properties at the limits between solid and liquid states. Moreover all these unique features might deserve to confirm a number of structural descriptors explaining the structure-directed functions of natural channel proteins. The water-free I-quartet-"off form" superstructure described by our group is reminiscent of a closed proton gate conformation of the M2 Influenza A M2IA protein [12, 13]. Slight conformational changes lead to the water assisted opening of the I-quartets through which protons can diffuse along dipolar oriented water-wires in the open state pore-gate region. Our opinion is that the I-quartet system represents a fundamentally important biomimetic superstructure for water/proton transport that can be extensively developed based on new structural motifs.

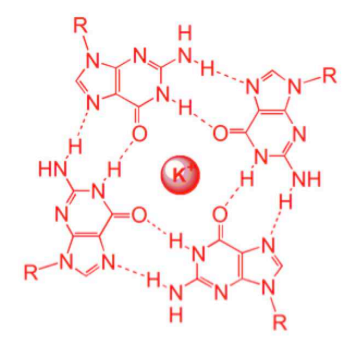

G-quartet

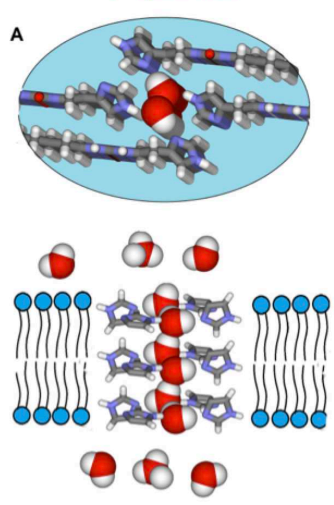

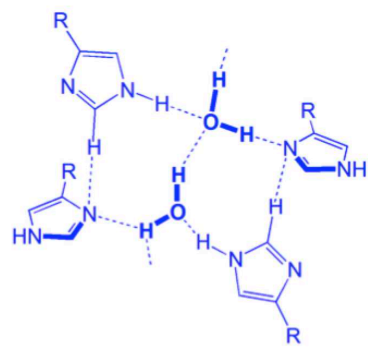

I-quartet

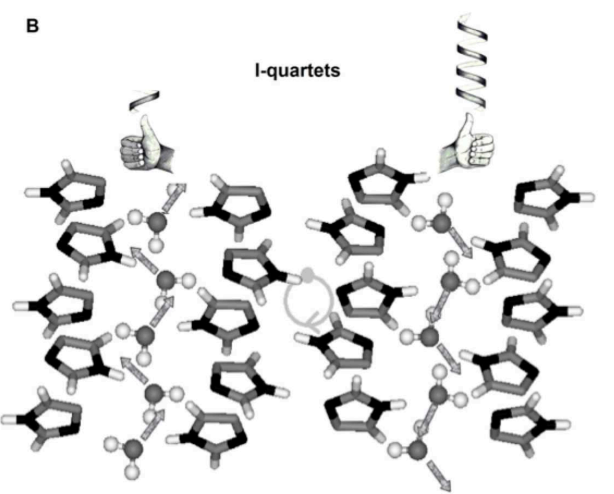

Fig. 2. Cross section and top views of Imidazole I-quartets generating water-channels in which the water molecules present a unique dipolar orientation. The I-quartets presenting supramolecular chirality, can accommodate dipolar water-wires along the length of the channel. Water molecules are shown either in van der Waals or in ball and stick representation.

First results may illustrate these ideas. Previous MD simulation studies of dynamic behaviors of the artificial I-quartet water channels and water molecules under confined conditions in a lipid bilayer environment were reported in [19-21]. An important aspect to bear in mind from these works is the quantitative correlation be- 
tween the dipolar orientation of water-wires and their effects on their stability once inserted in the bilayer membranes. Water and proton translocation properties through AWCs are interlinked with the water-wire orientation as well. Two extreme cases are illustrated in Fig. 3, where water is either permeating in a random pathway or through highly ordered channels, depending on small modifications on the I-quarted building blocks. These studies provide detailed experimental data correlated with extensive theoretical simulations on water permeability with efficiency similar to that of natural biological systems. The theoretical stability and dynamics of the assembly have been tested first, probing different stoichiometries and box sizes. As next steps, the energetics of water transport will be assessed [54]. This knowledge will enable us to suggest modifications in the channel scaffold to modulate and improve water transport that can subsequently be correlated experimentally. Once a full characterization at an all-atom level has been achieved, we will be able to devise a simplified coarse-grained model in order to study larger assemblies and longer timescales.

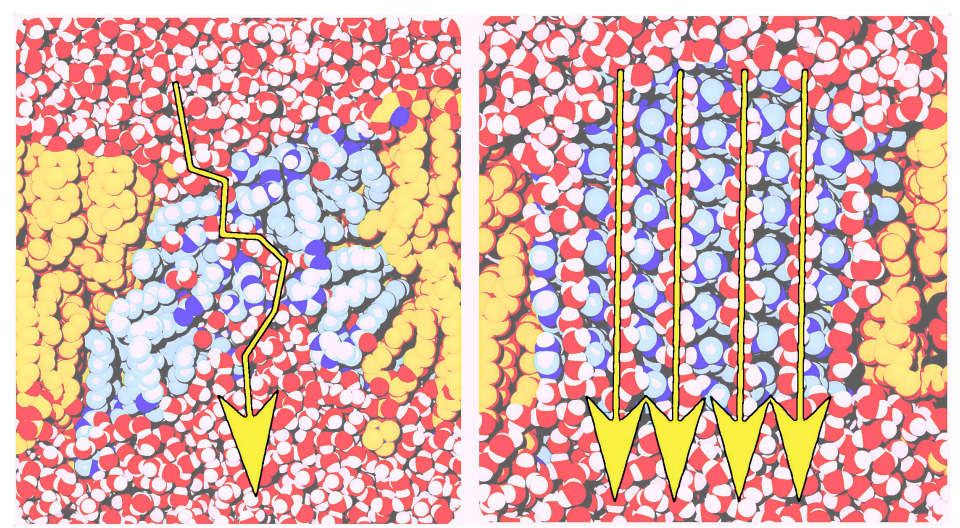

Fig. 3. Sponge-like and ordered water transport mechanisms observed in I-quartet channels [21].

\section{Computational Design}

\section{A roadmap for improving our current understanding}

Here we sketch a computationnally-focused approach exploring unpaved ways to design artificial systems for water transport in bilayer membranes closer to the natural ones with the goal to further improve AWC performance. Their resultant functionality can be dependent on the nature of the scaffold and added functionalities, as their multivalent interactions have been shown to be influenced by such factors as nature of the confining matrix, the stability and the orientation of the 
channel, etc. In this context, computational studies, rational design and engineering of water in biological systems fulfils the following aims:

- Improve the understanding of the adaptive functional self-organization of molecular components into functional channel platforms by using a self-adaptive strategy and by exploring the synergistic influence of structural selfassembly/platform function and of the evolutive behaviours on the addition of different external stimuli.

- Accurately control functional responses through bilayer membrane platforms developed via tuned incorporation of components into channel superstructures

- Investigation of application avenues towards larger impact of developed bioand environmental responsive materials for reverse osmosis (RO) and forward osmosis (FO) membranes.

Such an approach is challenging and ambitious but highly feasible since, although based on new supramolecular/constitutional/MD simulation concepts, it relies on substantial preliminary data, expertise, experience and facilities related in particular to the I-quartet channel family of compounds illustrated in Figs. 1b and 2. Molecular dynamics simulations strongly contribute to understand and improve the dynamic behaviors of water molecules under confined conditions. The key is to fine-tune the balance between attractive and repulsive elements. The formation of single-file columns of water confined along the internal pore surfaces is a critical element. It may impose a net-dipole alignment of water molecules. Such an alignment influences the conduction of fluids, envisioned to diffuse along the dipolar hydrophilic pathways. Synthetic architectures generating stable water/proton translocation pathways in bilayer membranes to mimick the natural protein functions can be characterized at high spatial- and time resolutions. The dynamic features of these objects confined in a lipid bilayer create new opportunities to further modify and engineer such systems.

Ideally, the supramolecular water-channels to be synthesized would require a nearly frictionless diffusional pathway of the water on the length scale of the pore trajectory. What is needed is an enhanced liquid flow mechanism at the location of artificial pores - one critical hallmark for mimicking protein channels - or doing better. The strong interactions of the water with the inner surface of the channels reduce the efficiency of water transport, when compared with the natural systems. However, understanding the fine details of the water transport mechanism at the microscopic level is essential to decipher how hydrogen-bond switch events can assist efficient in-pore diffusivity. Straightforward synthetic access to superstructures - artificial Aquaporins - allowing water wires give rise to novel strategies to constitutionally build up very selective water transport devices.

Despite the few advances that have been made in this very young field, one may say that the discovery of novel synthetic systems able to form water-channels is most of the time empirical. But even if the discovery is empirical, the natural proteins may serve us to tailor the functions of the synthetic systems and engineer optimized molecular constructs. This interdependence makes our next challenge still 
more ambitious: understanding the natural systems transport mechanism is often one way to raise artificial systems to the level of natural functions or even beyond. Then, if we can imagine how the system is designed in our hands (minds) we could ask a number of questions. For this purpose it is particularly important to form structural models in our minds. Advanced visualization is a particularly powerful approach already extensively used to understand biological membrane objects [55]. We will get back to the strengths of novel technological approaches such as using virtual reality gear or high-resolution and large-scale display walls a little later in this discussion [56-60]. The aim of such a visual analysis is to bring novel concepts to the engineering of nanostructured functional systems, together with the understanding of the mechanisms underlying a rational use of water transport functions employed as the nanostructuring tools. Such an understanding would contribute both to fundamental and applicative research. For the former, leading to a better understanding of the natural function of protein channels and solving important biological scenarios of oriented water diffusion along channels. For the latter, learning form natural water purifying systems we might shed light on new strategies to generate highly selective materials/systems for advanced water purification.

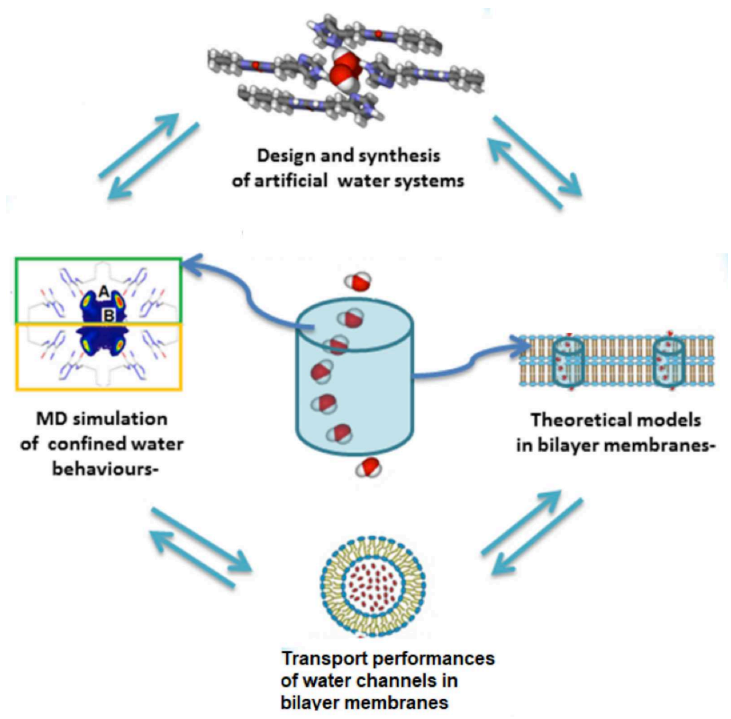

Fig. 4. illustrates the interplay of synthesis, experimental characterization and computational modeling. Water channel systems are synthetically engineered to enable platforms for the selective transport of water with active molecular control and motion. MD simulations and theoretical models of the channels embedded in realistic membrane systems guide the design of active systems through a mechanistic understanding of both the self-assembly process and the transport characterization offering experimental verification of the modeling predictions. Such an undertaking requires theoretical molecular models in realistic lipid bilayers and polymers, the design, synthesis and facilitated transport of water channel systems, and the structural characterization, stability studies of AWCs through bilayer membranes. 
The proposed approach, fundamental in nature, should permit to develop not only new knowledge but also to gain a greater insight on Biomimetic Artificial Water Systems, which can serve to understand complicated biological scenarios or to imagine novel membrane systems for water purification.

\section{Theoretical molecular models in realistic lipid bilayers and polymers}

A computational pipeline permits to explore the molecular organization of the candidate molecules in the membrane, and elucidate the mechanism of water transport. The approach we envision is based on coarse-grained (CG) and atomistic simulations supported by enhanced sampling. For a given compound, MD simulations based on CG models will be carried out so to explore and to individuate via enhanced sampling the possible free energy minima of the aggregates in the membrane. Several initial conditions should be tested (crystal like arrangement, spontaneous early steps aggregation, human design) so to enlarge the exploration. The individuated most stable aggregated states are filtered according to different criteria (energetics, packing, percolation) and will be back-mapped to the atomistic resolution. Further MD will be performed to test their capability for water transport and ion rejection. For the first study-cases the enhanced sampling will be performed at atomistic resolution to control the possible biases of the CG preliminary screening. The workflow will be performed for several stoichiometries. For each compound the combined information from the theoretically deduced structural/functional response of the molecules and from experiments, will suggest molecular modifications to be submitted to the pipeline depicted in Fig. 4. The preliminary CG approach has the advantage to reduce the computational cost for the most time-consuming part of the pipeline, the sampling of self-assembled structures. Such an approach should answer the following main questions:

- what is the structure and stability of AWCs in a membrane (or polymer) environment?

- how do water transport and ion rejection happen at the molecular level?

- how can transport (and AWCs properties in general) be tuned and optimized?

To further understand the structure and stability in a membrane environment, we need to investigate how the channels (self)assemble. The self-aggregation process raises a number of questions. What is the size of the aggregates? Are all channels identical/homogeneous? How does chirality affect the process?

To address water-channel functionality in a saline condition, we need to describe how precisely transport happens. MD simulations may be used to systematically examine the transport through AWCs of novel materials and specific AWCs to elucidate the role of hydration and detailed molecular interaction during nanofiltration. What is the geometry, mechanism and energetics of the process? How are 
ions rejected, and what happens to protons? These are essential characteristics that will enable to proceed to rational optimization of AWCs.

As briefly mentioned above, we performed MD simulations to understand the structure/activity relationships of the artificial water channels formed by Iquartets. We embedded small (ca. $3 \mathrm{~nm}$ wide) I-quartet channel patches based on the X-ray structures in a lipid bilayer environment with the same composition as that used in the experiments to begin each simulation. The MD results showed that I-quartets preferentially located within the bilayer membrane region and stabilized water channels in all simulations, although the degrees of water-occupancy, transport and structuring varied. We observed that the structural dynamics of Iquartet channels was dependent on the lateral pressure applied to the membrane, with higher pressure favoring the structuring of the water-wires and stabilizing the structure of the I-quartets. Very interestingly, different water permeation mechanisms were observed ranging from disordered cross-membrane pathways to strongly regular channel structures as depicted in Fig. 3.

Water transport and ion rejection characterization can be addressed even more quantitatively through Potential-of-Mean-Force type approaches or use of adapted collective variables to enhance the sampling. Osmotic gradients may be used to mimic experimental conditions. These techniques enable to determine quantitative energetics for transport of water, proton and ionic species. Statistical analysis can then provide the key features (geometry, mechanism) of transport. Large(time\&length)scale assessments may be required for selected systems, incorporating effects such as hydrodynamics within a simplified model.

Tuning of AWC properties to optimize transport and stability may be treated in an iterative fashion. First one may investigate a number of modifications such as decorating the transport channel walls. Such approaches were successfully used for understanding ion channel properties (work by Beckstein and Sansom, for instance $[33,34,37,61])$ as well as e.g. water transport through carbon nanotubes (work by Hummer $[35,36]$ ). We can draw on our own experience with ion channels and the fine characterization of water in biological systems [54]. Each modification should then be analyzed in terms of transport efficiency, ion rejection capability and stability.

\section{Design, synthesis and facilitated transport of water channel systems}

The key to the design of water channels is having stable pores wider than the critical diameter for selective binding to allow free flow of the water molecules. In general two main water translocation mechanisms were identified in selective filter channels working on a size restriction. They are related to:

- hydrophobic pores in which no H-bonding are occurring between the water and the channels and 
- hydrophilic pores that specifically bind water through hydrogen-bonds, to the wall of the channel.

In the first case the design might be fairly straightforward; the size of the hydrophobic pore is needed to be $<3 \AA$ to block the passage of hydrated ions. This difficulty still lies in the synthetic routes for obtaining hydrophobic channels without H-bonding moieties that might induce the selective transport of water against the ions. Whilst the size restriction is necessary, water specific binding is needed for selectivity. We already find important structural details toward this goal, because of the recent discovery by our group of the artificial I-quartet channels introduced previously, having $100 \%$ selectivity against salts and very interesting water permeability of $\sim 1.5 \times 10^{\circ}$ water molecules/second/channel, approaching those of natural systems $[7,26]$. To the best of our knowledge, the I-quartets are the only channels nowadays presenting total ion-exclusion, based on dimensional steric reasons, whereas water clustering effects appear to be also important [26]. The selective binding of water can be achieved through the synergetic combination of donor-acceptor H-bonding moieties. In both cases binding/transport of cations or anions are prohibited, as such processes would induce an energy penalty from the dehydration of the ions due to the simultaneous donor-acceptor interactions with the hydrogen-bonding channels, mainly leading to the selectivity of the water in competition with other ions.
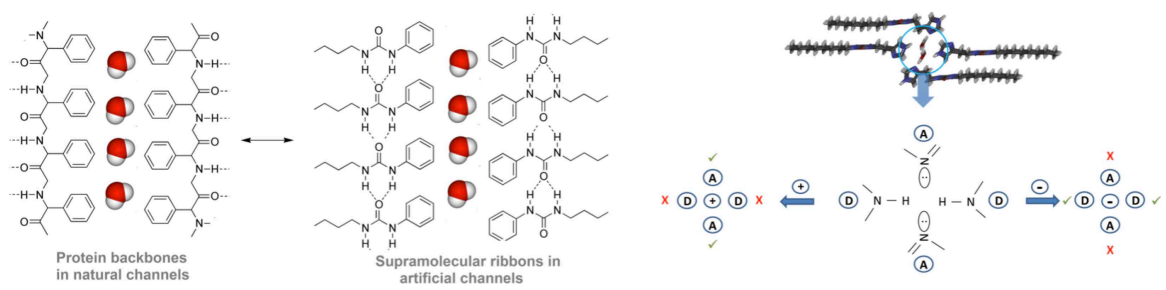

Fig. 5. Biomimetic artificial water channels (left) Natural protein versus artificial supramolecular ribbon self-assembly for water channel generation (right). Donor-acceptor-donor-acceptor Hbonding moieties in donor-acceptor based channels for water recognition incompatible with the binding of cations and anions.

We now intend to reach the next goal of making more competitive channels with controlled transport properties. This objective may require novel synthons combining a particularly broad range of features, decorating the functional polar head with various functionalities in order to enhance self-assembly and to control water binding properties of the polar head. The directional supramolecular guiding $\mathrm{H}$ bonding interaction is reminiscent of the amide moiety's H-bonding in proteins and can be varied in order to modulate the dynamics of the ribbon's self-assembly. The hydrophobic tail provides another design opportunity. 


\section{Advanced visual exploration}

\section{Spatial complexities in visually understanding AWCs}

Analyzing water channels through membrane-traversing pores on a $2 \mathrm{D}$ standard desktop is a complex task due to the lack of a true perspective view of the spatial structure. Such channels may form intricate three-dimensional ramifications within the membrane. The difficulty resides in identifying precisely and effectively which part of the I-quartet molecules interacts with the water and constitute transport channels. In the models we investigated, several close-by yet independent channels are present, hence one may easily misinterpret which water molecule belongs to which channel in a typical crowded 2D projection. More advanced and intrinsically three-dimensional display hardware solutions provide powerful alternatives with respect to $2 \mathrm{D}$ screens to address these issues. We have carried out first experiments with three different setups, comparing a 2D desktop environment, virtual reality headsets for truly immersive in-depth exploration of artificial water channels, as well as a large-scale high-resolution display wall. The VR approach is very powerful to immerse an individual into the molecular world, whereas the display wall is more naturally prone to collaborative exchanges around a complex 3D scene. We use our open-source UnityMol software [62-64] for all experiments described here. The software is available for download and operates in desktop (2D), display wall (3D) and virtual reality headset (immersive $3 \mathrm{D}$ with head-tracking) modes.

\section{Navigating through AWCs in three dimensions}

First experiments using 3D glasses on the display wall immediately showed the gain of adding a 3 rd dimension to the visual exploration. With respect to the water channel visualization, the $3 \mathrm{rd}$ dimension adds depth of field enabling a scientist to more easily identify each channel and to highlight the water molecules present in the interior of a given pore. The large size of the display makes it easy to unite several collaborators with complementary expertise such as a domain scientist, an expert in visualization, a molecular modeling professional etc., such as to optimize the process and jointly achieve the best analysis and visual inspection of the system. In particular when using a surface representation of the channels and pores the $3 \mathrm{D}$ visualization provides a strong gain through improved perception of bumps and crevices. By (arbitrarily and geometrically) dividing the channels in two halves and displaying them as surfaces with different attributes, for instance in terms of color or transparency, the visualization is improved and the identification of differences in stabilization and permeation on each "side" is facilitated. Using a 
cutting plane to expose the channel interior is another approach that works well. When using the virtual reality headset instead, such a visual system preparation becomes less important because one can easily move inside the molecule, finely control and change the point of view to adjust to a given channel of interest instead of moving the whole molecular system into focus. The visual experience is clearly enhanced and requires less preparation to be efficient when moving from a desktop to 3D display wall to VR headset context. In all three cases it has proven difficult to intuitively select interesting parts of the system without an advanced geometrical selection capability, as the pores do not follow a unique and easily identifiable direction in the coordinate system. A prior selection, for example based on relevant residue ids allows to work around this current limitation.
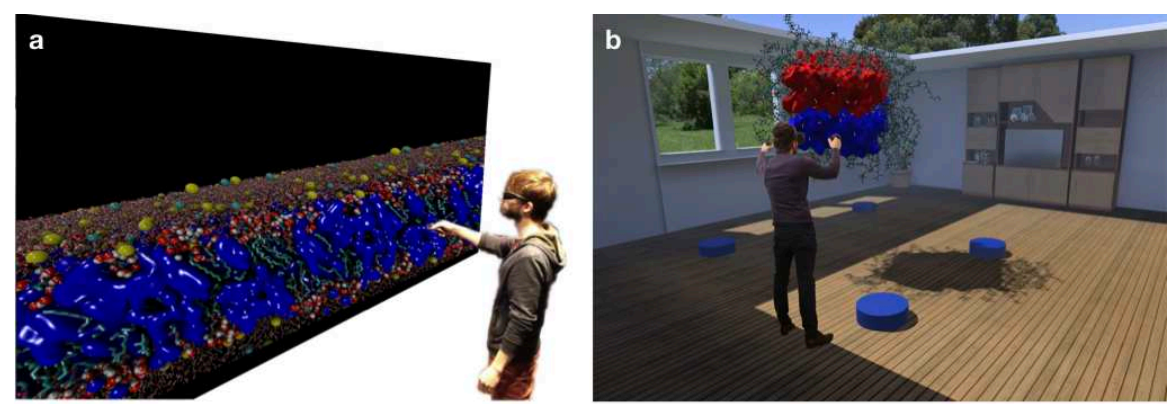

Fig. 6. Visual experiments exploring the properties of the I-quartet channel water conduction pathways on a 3D stereoscopic display wall (a) and in a fully immersive virtual reality environment using a headset (b). Both panels represent illustrative photomontages of such experiments.

\section{First user feedback}

Although the 3D display hardware capacities are clearly benefical to the exploration, they are by no means sufficient to obtain insight into these complex molecular systems. It is as necessary to finetune the visual representations as it is to add the third dimension. We have collected the first user feedbacks on avenues for improving the visual repertoire for these systems.

The lipid bilayer is a useful visual cue, however it easily saturates the visual channel. An abstract and simplified representation, such as for instance depicting the phosphorus atoms representing the lipid headgroups as a cue for the bilayer provides a better experience. Similarly, representing parts of the system through transparency is required to depict the context without hiding the water channels and not overloading the scene. Highlighting hydrogen bonds in the system further helps to identify stabilizing elements holding the water channels together. By depicting the surface blobs of the water channels throughout trajectory playback, it is easily possible to follow leakage or possibly breakdown of the channels. Such ob- 
servations naturally generate ideas about key parameters in the system and how to possibly quantify them. In the present case, for instance measuring the pore diameter during trajectory playback to quantify "openness". Some of these features still need to be implemented or refined in the UnityMol software before the next round of experiments can be carried out. Finetuning immersive visual analytic tools is a lengthy and iterative process of which we only have scratched the surface so far.

\section{Conclusion}

The field of artificial water-channels is still a very young field. High conduction activity obtained with natural compounds can be bio-mimicked using simpler compounds displaying constitutional functions like the natural ones in order to create all-made artificial biomimetic channels by using synthetic approaches. Improved synthetic biomimetic water-channels and pores bear great promise to enhance our insight into the natural function of macromolecular channels. Their development may lead to new strategies for the design of highly selective, advanced water purification systems. The membrane-assembled architectures, mimicking the natural protein functions and generating water/proton translocation pathways in bilayer membranes can be theoretically scrutinized to unravel underlying mechanisms. Because of the spatial complexities, advanced visualization approaches are particularly precious to achieve efficient visual analytics of these membranetraversing objects.

Acknowledgments. This work was conducted within the ANR-15-CE29-0009 DYNAFUN (Mihail Barboiu) and ANR-18-CE06-0004 WATERCHANNELS (Fabio Sterpone) projects. The study was further supported by the "Initiative d'Excellence" program from the French State (Grant "DYNAMO", ANR-11-LABX0011 and grant "CACSICE", ANR-11-EQPX-0008). X.M., A.H., H.S. and M.B. thank Sesame Ile-de-France for co-funding the display wall used in this study. Computational work was performed using HPC resources from GENCI-CINES (grant number 2016-072292) to Marc Baaden. X.M. and M.B. thank UCB Biopharma for support. This publication received support from the European Union's Horizon 2020 research and innovation programme under Grant No. 667387 WIDESPREAD 2-2014 SupraChem Lab.

1. Ball P (2008) Water as an Active Constituent in Cell Biology. Chem Rev 108:74-108 . https://doi.org/10.1021/cr068037a

2. Bellissent-Funel M-C, Hassanali A, Havenith M, Henchman R, Pohl P, Sterpone F, van der Spoel D, Xu Y, Garcia AE (2016) Water Determines the Structure and Dynamics of Proteins. Chem Rev 116:7673-7697 . https://doi.org/10.1021/acs.chemrev.5b00664

3. Gokel GW, Mukhopadhyay A (2001) Synthetic models of cation-conducting channels. Chem Soc Rev 30:274-286 . https://doi.org/10.1039/b008667n 
4. Sakai N, Mareda J, Matile S (2005) Rigid-Rod Molecules in Biomembrane Models: From Hydrogen-Bonded Chains to Synthetic Multifunctional Pores. Acc Chem Res 38:79-87 . https://doi.org/10.1021/ar0400802

5. Cazacu A, Tong C, van der Lee A, Fyles TM, Barboiu M (2006) Columnar Self-Assembled Ureido Crown Ethers: An Example of Ion-Channel Organization in Lipid Bilayers. J Am Chem Soc 128:9541-9548 . https://doi.org/10.1021/ja061861w

6. Fyles TM (2007) Synthetic ion channels in bilayer membranes. Chem Soc Rev 36:335-347 . https://doi.org/10.1039/B603256G

7. Le Duc Y, Michau M, Gilles A, Gence V, Legrand Y-M, van der Lee A, Tingry S, Barboiu M (2011) Imidazole-Quartet Water and Proton Dipolar Channels. Angew Chem Int Ed 50:11366-11372 . https://doi.org/10.1002/anie.201103312

8. Barboiu M (2012) Artificial Water Channels. Angew Chem Int Ed 51:11674-11676 . https://doi.org/10.1002/anie.201205819

9. Barboiu M, Gilles A (2013) From Natural to Bioassisted and Biomimetic Artificial Water Channel Systems. Acc Chem Res 46:2814-2823 . https://doi.org/10.1021/ar400025e

10. Barboiu M, Le Duc Y, Gilles A, Cazade P-A, Michau M, Marie Legrand Y, van der Lee A, Coasne B, Parvizi P, Post J, Fyles T (2014) An artificial primitive mimic of the Gramicidin-A channel. Nat Commun 5:4142 . https://doi.org/10.1038/ncomms5142

11. Barboiu M, Cazade P-A, Le Duc Y, Legrand Y-M, van der Lee A, Coasne B (2015) Polarized Water Wires under Confinement in Chiral Channels. J Phys Chem B 119:87078717 . https://doi.org/10.1021/acs.jpcb.5b03322

12. Schnell JR, Chou JJ (2008) Structure and mechanism of the M2 proton channel of influenza A virus. Nature 451:591-595 . https://doi.org/10.1038/nature06531

13. Hu F, Luo W, Hong M (2010) Mechanisms of Proton Conduction and Gating in Influenza M2 Proton Channels from Solid-State NMR. Science 330:505-508. https://doi.org/10.1126/science.1191714

14. Cheruzel LE, Pometun MS, Cecil MR, Mashuta MS, Wittebort RJ, Buchanan RM (2003) Structures and Solid-State Dynamics of One-Dimensional Water Chains Stabilized by Imidazole Channels. Angew Chem Int Ed 42:5452-5455 https://doi.org/10.1002/anie.200352157

15. Fei Z, Zhao D, Geldbach TJ, Scopelliti R, Dyson PJ, Antonijevic S, Bodenhausen G (2005) A Synthetic Zwitterionic Water Channel: Characterization in the Solid State by X-ray Crystallography and NMR Spectroscopy. Angew Chem Int Ed 44:5720-5725. https://doi.org/10.1002/anie.200500207

16. Legrand Y-M, Michau M, van der Lee A, Barboiu M (2008) Homomeric and heteromeric self-assembly of hybrid ureido-imidazole compounds. CrystEngComm 10:490 . https://doi.org/10.1039/b717015g

17. Legrand Y-M, van der Lee A, Masquelez N, Rabu P, Barboiu M (2007) Temperature Induced Single-Crystal-to-Single-Crystal Transformations and Structure Directed Effects on Magnetic Properties. Inorg Chem 46:9083-9089 . https://doi.org/10.1021/ic700867b

18. Barboiu M (2016) Artificial water channels - incipient innovative developments. Chem Commun 52:5657-5665 . https://doi.org/10.1039/C6CC01724J

19. Licsandru E, Kocsis I, Shen Y, Murail S, Legrand Y-M, van der Lee A, Tsai D, Baaden M, Kumar M, Barboiu M (2016) Salt-Excluding Artificial Water Channels Exhibiting Enhanced Dipolar Water and Proton Translocation. J Am Chem Soc 138:5403-5409 . https://doi.org/10.1021/jacs.6b01811

20. Kocsis I, Sorci M, Vanselous H, Murail S, Sanders SE, Licsandru E, Legrand Y-M, van der Lee A, Baaden M, Petersen PB, Belfort G, Barboiu M (2018) Oriented chiral water wires in artificial transmembrane channels. Sci Adv 4:eaao5603. https://doi.org/10.1126/sciadv.aao5603

21. Murail S, Vasiliu T, Neamtu A, Barboiu M, Sterpone F, Baaden M (2018) Water permeation across artificial I-quartet membrane channels: from structure to disorder. Faraday Discuss 209:125-148 . https://doi.org/10.1039/C8FD00046H 
22. Sterpone F, Derreumaux P, Melchionna S (2015) Protein Simulations in Fluids: Coupling the OPEP Coarse-Grained Force Field with Hydrodynamics. J Chem Theory Comput 11:1843-1853 . https://doi.org/10.1021/ct501015h

23. F. Brandner A, Timr S, Melchionna S, Derreumaux P, Baaden M, Sterpone F (2019) Modelling lipid systems in fluid with Lattice Boltzmann Molecular Dynamics simulations and hydrodynamics. Sci Rep 9:16450 . https://doi.org/10.1038/s41598-019-52760-y

24. Agre P (2004) Aquaporin Water Channels (Nobel Lecture). Angew Chem Int Ed 43:4278-4290 . https://doi.org/10.1002/anie.200460804

25. Tajkhorshid E (2002) Control of the Selectivity of the Aquaporin Water Channel Family by Global Orientational Tuning. Science 296:525-530 https://doi.org/10.1126/science.1067778

26. Kumar M, Grzelakowski M, Zilles J, Clark M, Meier W (2007) Highly permeable polymeric membranes based on the incorporation of the functional water channel protein Aquaporin Z. Proc Natl Acad Sci 104:20719-20724 . https://doi.org/10.1073/pnas.0708762104

27. Hinds BJ (2004) Aligned Multiwalled Carbon Nanotube Membranes. Science 303:6265 . https://doi.org/10.1126/science. 1092048

28. de Groot BL, Grubmüller H (2005) The dynamics and energetics of water permeation and proton exclusion in aquaporins. Curr Opin Struct Biol 15:176-183. https://doi.org/10.1016/j.sbi.2005.02.003

29. Kaucher MS, Peterca M, Dulcey AE, Kim AJ, Vinogradov SA, Hammer DA, Heiney PA, Percec V (2007) Selective Transport of Water Mediated by Porous Dendritic Dipeptides. J Am Chem Soc 129:11698-11699 . https://doi.org/10.1021/ja076066c

30. Hu X-B, Chen Z, Tang G, Hou J-L, Li Z-T (2012) Single-Molecular Artificial Transmembrane Water Channels. J Am Chem Soc 134:8384-8387 https://doi.org/10.1021/ja302292c

31. Si W, Chen L, Hu X-B, Tang G, Chen Z, Hou J-L, Li Z-T (2011) Selective Artificial Transmembrane Channels for Protons by Formation of Water Wires. Angew Chem Int Ed 50:12564-12568 . https://doi.org/10.1002/anie.201106857

32. de Groot BL, Grubmüller H (2001) Water permeation across biological membranes: mechanism and dynamics of aquaporin-1 and GlpF. Science 294:2353-2357. https://doi.org/10.1126/science.1062459

33. Beckstein O, Sansom MSP (2004) The influence of geometry, surface character, and flexibility on the permeation of ions and water through biological pores. Phys Biol 1:42-52 https://doi.org/10.1088/1478-3967/1/1/005

34. Rao S, Lynch CI, Klesse G, Oakley GE, Stansfeld PJ, Tucker SJ, Sansom MSP (2018) Water and hydrophobic gates in ion channels and nanopores. Faraday Discuss 209:231-247 https://doi.org/10.1039/c8fd00013a

35. Hummer G, Rasaiah JC, Noworyta JP (2001) Water conduction through the hydrophobic channel of a carbon nanotube. Nature 414:188-190. https://doi.org/10.1038/35102535

36. Kalra A, Garde S, Hummer G (2003) Osmotic water transport through carbon nanotube membranes. Proc Natl Acad Sci U S A 100:10175-10180. https://doi.org/10.1073/pnas.1633354100

37. García-Fandiño R, Sansom MSP (2012) Designing biomimetic pores based on carbon nanotubes. Proc Natl Acad Sci U S A 109:6939-6944 https://doi.org/10.1073/pnas.1119326109

38. Barboiu M, Kumar M, Baaden M, Gale PA, Hinds BJ (2019) Highlights from the Faraday Discussion on Artificial Water Channels, Glasgow, UK. Chem Commun Camb Engl 55:3853-3858 . https://doi.org/10.1039/c9cc90112d

39. Baaden M, Barboiu M, Bill RM, Chen C-L, Davis J, Di Vincenzo M, Freger V, Fröba M, Gale PA, Gong B, Hélix-Nielsen C, Hickey R, Hinds B, Hou J-L, Hummer G, Kumar M, Legrand Y-M, Lokesh M, Mi B, Murail S, Pohl P, Sansom M, Song Q, Song W, Törnroth- 
Horsefield S, Vashisth H, Vögele M (2018) Biomimetic water channels: general discussion. Faraday Discuss 209:205-229 . https://doi.org/10.1039/c8fd90020e

40. Baaden M, Barboiu M, Borthakur MP, Chen C-L, Coalson R, Davis J, Freger V, Gong B, Hélix-Nielsen C, Hickey R, Hinds B, Hirunpinyopas W, Horner A, Hou J-L, Hummer G, Iamprasertkun P, Kazushi K, Kumar M, Legrand Y-M, Lokesh M, Mi B, Mitra S, Murail S, Noy A, Nunes S, Pohl P, Song Q, Song W, Tornroth-Horsefield S, Vashisth H (2018) Applications to water transport systems: general discussion. Faraday Discuss 209:389-414 . https://doi.org/10.1039/c8fd90022a

41. Baaden M, Barboiu M, Bill RM, Casanova S, Chen C-L, Conner M, Freger V, Gong B, Góra A, Hinds B, Horner A, Hummer G, Kumar M, Lokesh M, Mitra S, Noy A, Pohl P, Sadet A, Sansom M, Törnroth-Horsefield S, Vashisth H (2018) Structure and function of natural proteins for water transport: general discussion. Faraday Discuss 209:83-95 . https://doi.org/10.1039/c8fd90019a

42. Baaden M, Borthakur MP, Casanova S, Coalson R, Freger V, Gonzalez M, Góra A, Hinds B, Hirunpinyopas W, Hummer G, Kumar M, Lynch C, Murail S, Noy A, Sansom M, Song Q, Vashisth H, Vögele M (2018) The modelling and enhancement of water hydrodynamics: general discussion. Faraday Discuss 209:273-285 https://doi.org/10.1039/c8fd90021c

43. MacKinnon R (2004) Potassium Channels and the Atomic Basis of Selective Ion Conduction (Nobel Lecture). Angew Chem Int Ed 43:4265-4277 https://doi.org/10.1002/anie.200400662

44. Roux B (2002) Computational Studies of the Gramicidin Channel. Acc Chem Res 35:366-375 . https://doi.org/10.1021/ar010028v

45. Saparov SM, Pohl P (2004) Beyond the diffusion limit: Water flow through the empty bacterial potassium channel. Proc Natl Acad Sci 101:4805-4809 https://doi.org/10.1073/pnas.0308309101

46. Tang CY, Zhao Y, Wang R, Hélix-Nielsen C, Fane AG (2013) Desalination by biomimetic aquaporin membranes: Review of status and prospects. Desalination 308:34-40 . https://doi.org/10.1016/j.desal.2012.07.007

47. Barbour LJ, Orr GW, Atwood JL (1998) An intermolecular (H2O)10 cluster in a solidstate supramolecular complex. Nature 393:671-673 . https://doi.org/10.1038/31441

48. Yoshizawa M, Kusukawa T, Kawano M, Ohhara T, Tanaka I, Kurihara K, Niimura N, Fujita M (2005) Endohedral Clusterization of Ten Water Molecules into a "Molecular Ice" within the Hydrophobic Pocket of a Self-Assembled Cage. J Am Chem Soc 127:2798-2799 . https://doi.org/10.1021/ja043953w

49. Görbitz CH (2007) Microporous Organic Materials from Hydrophobic Dipeptides. Chem - Eur J 13:1022-1031 . https://doi.org/10.1002/chem.200601427

50. Müller A, Bögge H, Diemann E (2003) Structure of a cavity-encapsulated nanodrop of water. Inorg Chem Commun 6:52-53 . https://doi.org/10.1016/S1387-7003(02)00679-2

51. Mitra T, Miró P, Tomsa A-R, Merca A, Bögge H, Ávalos JB, Poblet JM, Bo C, Müller A (2009) Gated and Differently Functionalized (New) Porous Capsules Direct Encapsulates' Structures: Higher and Lower Density Water. Chem - Eur J 15:1844-1852 . https://doi.org/10.1002/chem.200801602

52. Esque J, Sansom MSP, Baaden M, Oguey C (2018) Analyzing protein topology based on Laguerre tessellation of a pore-traversing water network. Sci Rep 8:13540 . https://doi.org/10.1038/s41598-018-31422-5

53. Horner A, Pohl P (2018) Single-file transport of water through membrane channels. Faraday Discuss 209:9-33 . https://doi.org/10.1039/c8fd00122g

54. Sauguet L, Poitevin F, Murail S, Van Renterghem C, Moraga-Cid G, Malherbe L, Thompson AW, Koehl P, Corringer P-J, Baaden M, Delarue M (2013) Structural basis for ion permeation mechanism in pentameric ligand-gated ion channels. EMBO J 32:728-741 . https://doi.org/10.1038/emboj.2013.17 
55. Baaden M (2019) Visualizing Biological Membrane Organization and Dynamics. J Mol Biol 431:1889-1919 . https://doi.org/10.1016/j.jmb.2019.02.018

56. Hirst JD, Glowacki DR, Baaden M (2014) Molecular simulations and visualization: introduction and overview. Faraday Discuss 169:9-22 . https://doi.org/10.1039/c4fd90024c

57. Sommer B, Baaden M, Krone M, Woods A (2018) From Virtual Reality to Immersive Analytics in Bioinformatics. J Integr Bioinforma 15: . https://doi.org/10.1515/jib-2018-0043

58. Hirst, Johnson, Zheng, Rozmanov, Zoppè, Hall, O’Donoghue, Proctor, Glowacki, Delalande, Brancale, Baaden, Stone, Brooks, Olson, Chavent, Haag, Krone, Kouyoumdjian, Levieux, Reiher, Fowler, Raffin, Baaden, Montes, Dreher (2014) Virtual and augmented reality immersive molecular simulations: general discussion. Faraday Discuss 169:143-166 . https://doi.org/10.1039/c4fd90019g

59. Proctor, Ertl, Petrov, O’Donoghue, Brooks, Hirst, Stone, Krone, Zoppè, Hall, Baaden, Reiher, Sommer, Fowler, Rozmanov, Glowacki (2014) Advanced visualization and visual analytics: general discussion. Faraday Discuss 169:245-264 . https://doi.org/10.1039/c4fd90020k

60. Maes A, Martinez X, Druart K, Laurent B, Guégan S, Marchand CH, Lemaire SD, Baaden M (2018) MinOmics, an Integrative and Immersive Tool for Multi-Omics Analysis. J Integr Bioinforma 15: . https://doi.org/10.1515/jib-2018-0006

61. Beckstein O, Tai K, Sansom MSP (2004) Not ions alone: barriers to ion permeation in nanopores and channels. J Am Chem Soc 126:14694-14695 https://doi.org/10.1021/ja045271e

62. Lv Z, Tek A, Da Silva F, Empereur-mot C, Chavent M, Baaden M (2013) Game On, Science - How Video Game Technology May Help Biologists Tackle Visualization Challenges. PLoS ONE 8:e57990 . https://doi.org/10.1371/journal.pone.0057990

63. Doutreligne S, Cragnolini T, Pasquali S, Derreumaux P, Baaden M (2014) UnityMol: Interactive scientific visualization for integrative biology. In: 2014 IEEE 4th Symposium on Large Data Analysis and Visualization (LDAV). IEEE, France, pp 109-110

64. Laureanti J, Brandi J, Offor E, Engel D, Rallo R, Ginovska B, Martinez X, Baaden M, Baker NA (2020) Visualizing biomolecular electrostatics in virtual reality with UnityMolAPBS. Protein Sci Publ Protein Soc 29:237-246 . https://doi.org/10.1002/pro.3773 